\title{
Crossover Between Tilt Families and Zero Area Thermal Expansion in Hybrid Prussian Blue Analogues
}

\author{
A. E. Phillips* \\ A. D. Fortes ${ }^{\dagger}$
}

October 25, 2017

\begin{abstract}
Materials in the family of Prussian blue analogues $\left(\mathrm{C}_{3} \mathrm{H}_{5} \mathrm{~N}_{2}\right)_{2} \mathrm{~K}\left[\mathbf{M}(\mathrm{CN})_{6}\right]$, where $\mathrm{C}_{3} \mathrm{H}_{5} \mathrm{~N}_{2}$ is the imidazolium ion and $\mathbf{M}=\mathrm{Fe}$, Co, undergo two phase transitions with temperature: at low temperatures the imidazolium cations have an ordered configuration $(C 2 / c)$ while in the intermediate- and high-temperature phases (both previously reported as $R \overline{3} m$ ) they are dynamically disordered. We show from high-resolution powder neutron diffraction data that the high-temperature phase has zero area thermal expansion in the $a b$ plane. Supported by Landau theory and single-crystal X-ray diffraction data, we re-evaluate the space group symmetry of the intermediate-temperature phase to $R \overline{3}$. This reveals that the low-to-intermediate temperature transition is due to competition between two different tilt patterns of the $\left[\mathbf{M}(\mathrm{CN})_{6}\right]^{3-}$ ions. Controlling the relative stabilities of these tilt patterns offers a potential means to tune the exploitable electric behaviour that arises from motion of the imidazolium guest.
\end{abstract}

The Prussian blues, a family of materials in which metal ions are bound by cyanide ions into a cubic network, display a rich diversity of stoichiometry, structure, and functionality. A wide variety of metals may be incorporated into the framework or encapsulated in the interstices; depending on the stoichiometry, there may be defects in the framework which influence the porosity and magnetic properties. ${ }^{[1]}$ The exploitable properties of this family include ion sorption for nuclear waste cleanup, ${ }^{[2]}$ ion mobility for use as battery electrode

${ }^{*}$ Centre for Condensed Matter and Materials Physics, School of Physics and Astronomy, Queen Mary University of London, Mile End Road, London El 4NS, UK; a.e.phillips@qmul .ac .uk

${ }^{\dagger}$ ISIS Facility, Rutherford Appleton Laboratory, Chilton, Didcot, Oxon OX11 0QX, UK 
materials, ${ }^{[3,4]}$ guest uptake for use in sensors ${ }^{[5,6]}$ and photoswitchable magnetism. ${ }^{[7]}$ It is possible to prepare these materials as nanoparticles and thin films, and the properties can be tuned by varying both the composition and the morphology. ${ }^{[6,8]}$

Recently, new members of this well-studied family have been developed in which the cubic interstices are occupied by small organic cations. Similar to the well-known lead halide perovskite semiconductors, which are based on small ions such as methylammonium or formamidinium, Prussian blue analogues that encapsulate the slightly larger acetamidinium, guanidinium, dimethylammonium, and imidazolium ions have been reported. More generally still, these materials are part of the broader family of metal-organic perovskite analogues, in which possible linkers include the formate, azide, thiocyanate, and dicyanamide anions. ${ }^{[9-11]}$ Like the analogous perovskites, these materials tend to display multiple phases. The active distortion modes are often characterised by rigid rotations of the metal coordination octahedra, avoiding the energy penalty that would come from distorting them. However, the diatomic cyanide linker affords the network substantially more flexibility than the monoatomic linkers in the perovskites. A further difference from the inorganic perovskites comes from the organic guest cations, which may have intrinsic dipole or higherorder $^{[12]}$ multipole moments and often undergo different motion in different phases, leading to novel and exploitable electrical properties. ${ }^{[13,14]}$ The chemistry and physics of the inorganic perovskites depend directly on their structural distortions, and thus understanding the possible tilt modes has allowed specific functional properties to be engineered in these materials. If we are to develop similar control over the functional properties of the hybrid perovskites, it will be vital to understand the distortion modes of these materials, and the symmetry relationships between them, equally well.

We focus here on the imidazolium potassium hexacyanometallates, $\left(\mathrm{C}_{3} \mathrm{H}_{5} \mathrm{~N}_{2}\right)_{2} \mathrm{~K}\left[\mathbf{M}(\mathrm{CN})_{6}\right]$, where $\mathbf{M}=\mathrm{Fe}, \mathrm{Co}^{\left[{ }^{[15,16]}\right.}$ The structure of these materials is analogous to the double perovskites or elpasolites; it consists of rock-salt ordered potassium and hexacyanometalate ions, with imidazolium ions occupying the cubic pores in a structure. Each material undergoes two phase transitions with temperature. In the low-temperature phase (LTP), the imidazolium cations are frozen in an ordered configuration with space group $C 2 / c$. The intermediate- and high-temperature phases (ITP and HTP) have both previously been reported in space group $R \overline{3} \mathrm{~m}$. In these phases, the imidazolium cations are dynamically disordered about the threefold axis, giving an annulus of scattering density that can most easily be crystallographically modelled as a six-membered ring (Figure 1(a)).

If the HTP-ITP phase transition is indeed isosymmetric, Landau theory guarantees that it must be first-order. ${ }^{[17]}$ However, published DSC data show little sign of a latent heat associated with this transition. ${ }^{[15,16]}$ To investigate more closely, we performed high-resolution 
(a)

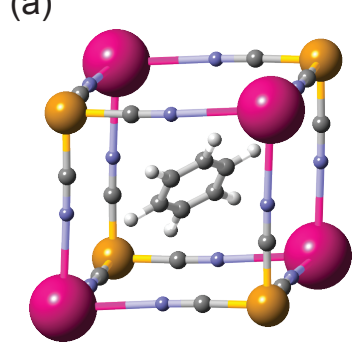

(c)

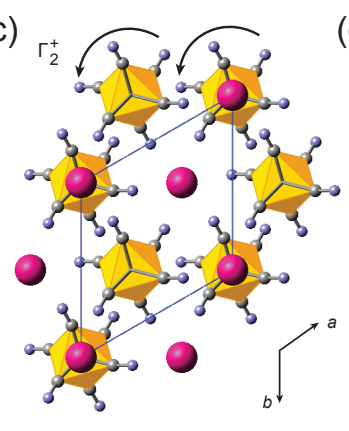

(b)

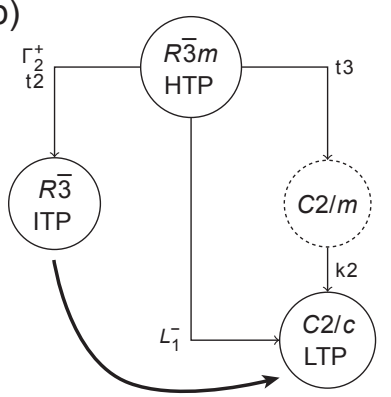

(d)

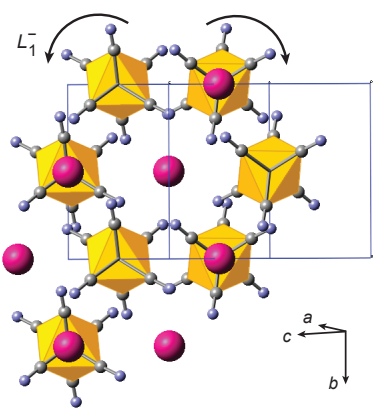

Figure 1: (a) Structure of the target materials, showing the crystallographic representation of the dynamically disordered imidazolium ion as a six-membered ring. Fe/Co atoms are shown in yellow, $\mathrm{K}$ in pink, $\mathrm{C}$ in grey, $\mathrm{N}$ in blue, $\mathrm{H}$ in white. (b) Groupsubgroup relationships between the three phases of this family of materials. The irreducible representation associated with each transition, order of the subgroup, and classification as klassengleiche or translationengleiche are is also indicated. For completeness, we include the hypothetical $C 2 / m$ structure that would result from losing the threefold symmetry without doubling the unit cell. The ITP-LTP transition on cooling involves a change of tilt system, indicated by the thick arrow. (c) In the intermediate-temperature phase, all hexacyanoferrate ions rotate in the same direction. (d) In the low-temperature phase, by contrast, the unit cell doubles and alternate hexacyanoferrate ions rotate in opposite directions. 
powder neutron diffraction measurements on perdeuterated samples of the Fe and Co materials using the HRPD instrument (ISIS, U.K.). Lattice parameters were determined by Rietveld refinement of the literature structures, and clearly show the two phase transitions at temperatures agreeing with published values (Figure 2(a,b)).

In the high-temperature phase, both compounds undergo anisotropic thermal expansion, with near-zero expansion in the $a b$ plane and strong positive expansion along the $c$ axis (Figure 2(a,b); Table 1). Anisotropic thermal expansion is well known in cyanide-bridged frameworks, often caused by a "wine-rack" effect whereby expansion in one direction is mechanically linked to contraction in perpendicular directions. ${ }^{[18,19]}$ By contrast, the combination of strong thermal expansion in one direction with little perpendicular contraction (Table 1) is much rarer.

This behaviour can be understood by transforming to a rhombohedral supercell, chosen to give a unit cell equivalent to the ideal $F m \overline{3} m$ Prussian blue aristotype (Figure 2(c,d)). This rhombohedral $a$ axis displays modest positive thermal expansion. This is nonetheless greater than the $a$ thermal expansion for most inorganic Prussian blues containing hexacyanoferrate(III) or hexacyanocobaltate(III) ions, ${ }^{[20,21]}$ suggesting that the large imidazolium guests in our materials block modes that might otherwise cause negative thermal expansion. ${ }^{[22,23]} \mathrm{By}$ contrast, the rhombohedral $\alpha$ angle, which we take to be obtuse, decreases with temperature towards the ideal cubic value of $90^{\circ}$. This is expected since the higher angle at lower temperature better accommodates the flat imidazolium ion; it is also in agreement with the empirical observation that metal-organic perovskite analogues with oblate A-site cations tend to have negative coefficients of thermal expansion with respect to the generalised $\alpha$ angle. ${ }^{[24]}$ Thus the near-zero area thermal expansion in the hexagonal $a b$ plane arises from the mutual cancellation of the rhombohedral $a$ expansion and $\alpha$ contraction. This immediately suggests a strategy for searching for more framework materials with thermal expansion exclusively along one axis: to identify framework materials with hexagonal crystal structures and planar (oblate) guest cations, such as imidazolium, guanidinium, or acetamidinium.

At the HTP-ITP transition, all lattice parameters changed continuously. Indeed, the transition is barely noticeable from the rhombohedral $a$ parameter (Figure 2(c,d)). The intermediate-to-high transition is thus clearly not first-order, but continuous. This implies that the space group of at least one of the two phases has been incorrectly assigned.

We investigated this further by laboratory single-crystal X-ray diffraction measurements on protiated samples of both materials in the ITP. In each case we found that the structure was best described in space group $R \overline{3}$, with the bridging cyanide ions displaced slightly away from the high-temperature mirror plane (Figure 2(e,f)). The $R \overline{3}$ structure refinements incorporated a twin law corresponding to reflection in the lost mirror plane. 


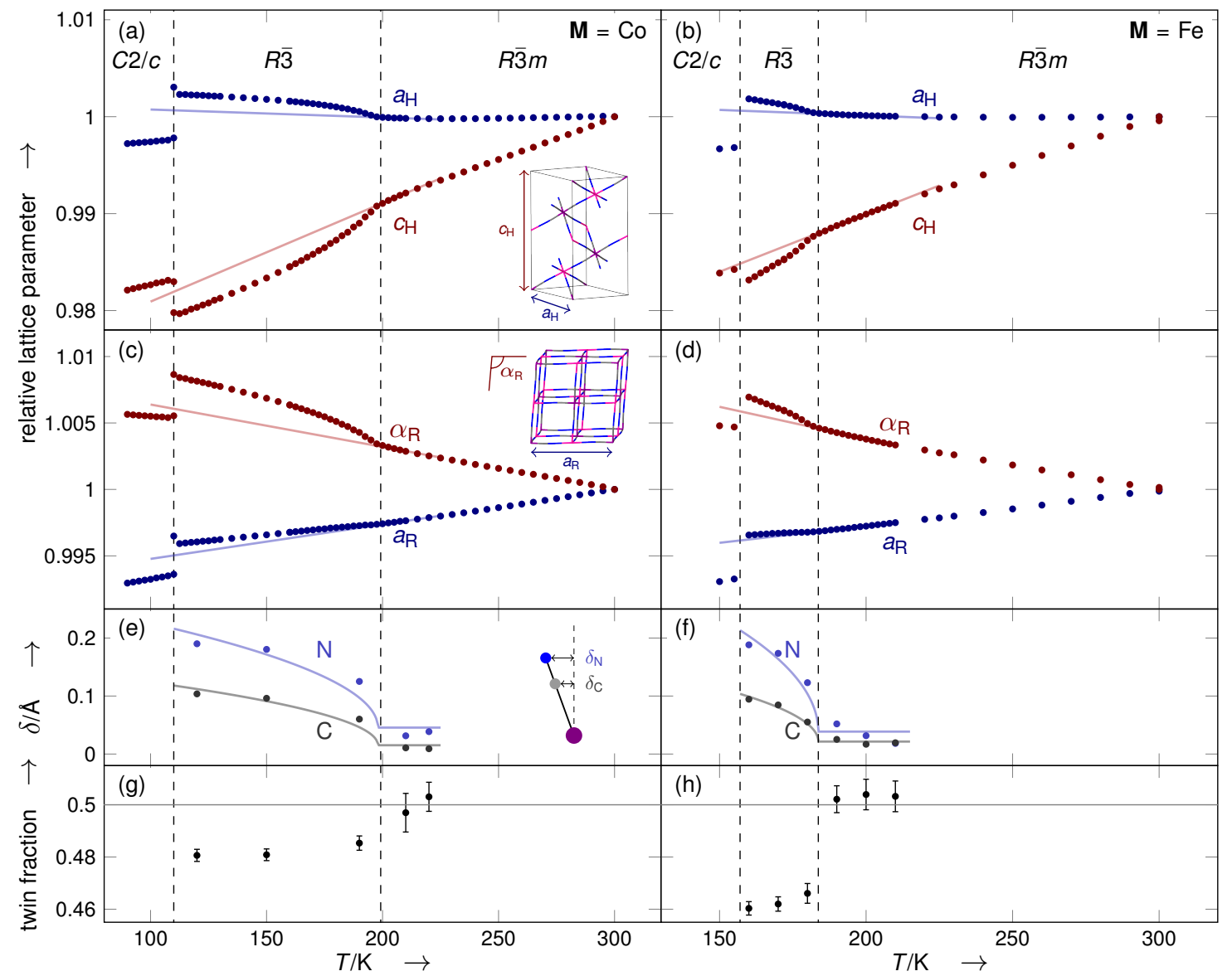

Figure 2: (a-d) Lattice parameters (HRPD) of the Co (left) and Fe (right) material as a function of temperature, relative to their values at $300 \mathrm{~K}$, referred to (a, b) hexagonal; (c, d) rhombohedral axes. Solid lines show linear fits to the lattice parameters immediately above the $R \overline{3}-R \overline{3} m$ phase transition. (e,f) Deviation $\delta$ of the cyanide C and N atoms from the lost mirror plane (SCXRD). Solid lines show fits of the expected form $\propto \sqrt{T_{\mathrm{c}}-T}$ plus a constant offset, but are intended principally as guides to the eye. (g, h) Fraction of one twin component (SCXRD). Data presented in (e-h) are based on $R \overline{3}$ refinements even in the $R \overline{3} m$ phase, to demonstrate the correct phase identification; see discussion in text. In all cases, unless error bars are explicitly shown, estimated standard deviations are smaller than the points used. 


\begin{tabular}{llcl}
\hline $\mathbf{M}$ & & $\mathrm{Co}$ & $\mathrm{Fe}$ \\
\hline$\alpha_{a_{\mathrm{H}}}=\partial a_{\mathrm{H}} / a_{\mathrm{H}} \partial T$ & $/ \mathrm{MK}^{-1}$ & $1.7(4)$ & $-1.3(3)$ \\
$\alpha_{c_{\mathrm{H}}}=\partial c_{\mathrm{H}} / c_{\mathrm{H}} \partial T$ & $/ \mathrm{MK}^{-1}$ & $87.3(4)$ & $99.8(2)$ \\
$\alpha_{a_{\mathrm{H}}} / \alpha_{c_{\mathrm{H}}}$ & & $0.020(4)$ & $-0.013(3)$ \\
\hline$\alpha_{a_{\mathrm{R}}}=\partial a_{\mathrm{R}} / a_{\mathrm{R}} \partial T$ & $/ \mathrm{MK}^{-1}$ & $25.9(2)$ & $27.3(3)$ \\
$\alpha_{\alpha_{\mathrm{R}}}=\partial \alpha_{\mathrm{R}} / \alpha_{\mathrm{R}} \partial T$ & $/ \mathrm{MK}^{-1}$ & $-31.9(3)$ & $-37.6(16)$ \\
\hline
\end{tabular}

Table 1: Coefficients of thermal expansion of the high-temperature phases referred to the hexagonal and rhombohedral cells, all taken as an average over the region $200 \mathrm{~K}$ to $300 \mathrm{~K}$.

It is not possible to distinguish between $R \overline{3}$ and $R \overline{3} m$ on the basis of $R$-factors alone. Refinement against the same data would invariably be expected to give lower $R$-factors in the lower-symmetry space group, simply because the number of parameters in that model is higher. Indeed, it was possible to refine an $R \overline{3}$ model even against HTP data. Our phase identification, however, is strongly supported by two features of the data. First, consider the refined displacement of the cyanide atoms from the lost mirror plane in the $R \overline{3}$ model (Figure 2(e,f)). This decreases on heating in the ITP, as one might expect from a displacement that effectively acts as an order parameter for this phase transition. Above the phase transition, in the HTP, the displacement reaches a low, roughly constant value, suggesting that at this point we are simply fitting to noise. Second, consider the refined fraction of the two twin components (Figure 2(g,h)). In the ITP, there was a small but measurable excess of one twin component, constant for a given sample. In the HTP, however, the twin fraction refined to exactly $50 \%$. In other words, in the ITP there was a measurable difference in the measured intensities of diffraction peaks related by the missing mirror plane, whereas in the HTP there was no such difference.

Further indirect support for this assignment comes from closely related structures that also crystallise in $R \overline{3}$. These include the inorganic analogue $\mathrm{Na}_{2} \mathrm{Mn}\left[\mathrm{Mn}(\mathrm{CN})_{6}\right]^{[25]}$ and the formate-bridged compounds $\left(\left(\mathrm{CH}_{3}\right)_{2} \mathrm{NH}_{2}\right)_{2} \mathrm{Na}\left[\mathbf{M}\left(\mathrm{HCO}_{2}\right)_{6}\right], \mathbf{M}=\mathrm{Al},{ }^{[26]} \mathrm{Cr} .{ }^{[27]}$

We now consider the implications of this revised space group assignment for the overall phase diagram of this series of materials. The ITP-LTP phase transition no longer involves a group-subgroup relationship. In the ITP the hexacyanometallate ions all rotate in the same direction, while in the LTP alternating planes rotate in opposite directions (Figure 1). The first-order ITP-LTP transition arises from competition between these two different tilt modes.

This behaviour contrasts strongly with the inorganic Prussian blues $\mathrm{Cs}_{2} \mathbf{M}^{\mathrm{I}} \mathbf{M}^{\mathrm{III}}(\mathrm{CN})_{6}, \mathbf{M}^{\mathrm{I}}=$ $\mathrm{Li}, \mathrm{K}, \mathrm{Rb} ; \mathbf{M}^{\mathrm{III}}=\mathrm{Cr}, \mathrm{Fe}, \mathrm{Co}$, Ir, which distort from a $F m \overline{3} m$ structure at high temperature to $P 2_{1} / n$ on cooling, in some cases going through an intermediate $P 4 / m n c$ phase. ${ }^{[28]}$ The two 
lower-symmetry phases are part of the same tilting sequence, different from that observed here. ${ }^{[29]}$

One important consequence of the polyatomic linker anions in the hybrid perovskite analogues is the possibility of activating "forbidden" distortion modes, which occur at Brillouin zone points where it would be impossible to distort an inorganic perovskite with monoatomic linker. ${ }^{[30]}$ Neither of the modes discussed here is "forbidden" in this sense. The $R \overline{3}$ structure, corresponding to a tilt pattern of $a^{-} a^{-} a^{-}$in Glazer notation, is included in systematic tabulations of the possible tilt modes of conventional double perovskites, and indeed is relatively common among them. ${ }^{[29,31]}$ The $C 2 / c$ structure, on the other hand, necessarily involves distortion of the $\mathrm{KN}_{6}$ octahedra, and hence cannot be represented by octahedral tilts alone in either conventional or hybrid perovskites. Perhaps for this reason, this structure is far rarer. To our knowledge, the only reported examples are the family $\mathrm{Pb}_{2} \mathbf{M S b O}_{3}, \mathbf{M}=$ $\mathrm{Y}, \mathrm{Tm}, \mathrm{Ho}, \mathrm{Er}, \mathrm{Yb}, \mathrm{Lu},{ }^{[32-34]}$ and $\mathrm{Pb}_{2} \mathrm{MnTeO}_{3} \cdot{ }^{[35] 1}$ Of these materials, only $\mathrm{Pb}_{2} \mathrm{MSbO}_{3}, \mathbf{M}=$ $\mathrm{Tm}, \mathrm{Yb}, \mathrm{Lu}$ also exhibit a $R \overline{3}$ phase. This observation is important for two reasons. First, it demonstrates that allowing forbidden modes is not the only significant effect of the cyanide framework's increased flexibility. Even considering only modes that are known in inorganic analogues, the framework flexibility decreases the energetic difference between distortion modes, making different modes accessible and competition between different sequences more likely. Second, the phase behaviour of the double perovskites has been attributed to the asymmetry of the $\mathrm{Pb}^{2+}$ cation, which has a sterochemically active lone pair. ${ }^{[32]}$ This result thus intriguingly suggests that the anisotropic imidazolium cation is in some sense mimicking the shape of the lead cation, which may be a promising avenue to explore in the development of lead-free functional materials.

To summarise, we have reexamined the structure of the title compounds using a combination of high-resolution neutron powder diffraction and single-crystal X-ray diffraction, revealing several remarkable structural properties that result from the flexibility of the cyanide framework and the anisotropy of the imidazolium guest cation. The high-temperature phase displays thermal expansion exclusively along the $c$ axis due to the competition between the expansion of the $\mathrm{M}-\mathrm{CN}-\mathrm{K}$ links and the distortion of the framework towards an ideal cubic structure. We have reanalysed the intermediate-temperature phase in space group $R \overline{3}$, showing that its structure involves a displacement of the cyanide atoms away from the lost mirror plane. This reassignment shows that the ITP and LTP involve different, competing tilt systems. These are not the same distortion modes as in the inorganic Prussian blues, but they are also found in a few double perovskites with A-site lead cations. Our results illustrate

${ }^{1}$ The chlorides $\mathrm{Cs}_{2} \mathrm{KInCl}_{3}$ and $\mathbf{A}_{3} \mathrm{BiCl}_{3}, \mathbf{A}=\mathrm{Cs}$, Rb have a more complicated structure with the same space group. 
that the combination of a flexible framework with an anisotropic guest can lead to unusual structural and thermodynamic properties that are otherwise difficult to achieve; such materials thus represent a useful strategy in the crystal engineering toolbox. In particular, we suggest that controlling the relative stabilities of these tilt patterns offers a potential means to tune the electric functionality that arises from the motion of the imidazolium guest.

We thank ISIS Neutron and Muon Source for the award of beam time.

\section{References}

[1] H. Tokoro, S. Ohkoshi, Dalton Trans 2011, 40, 6825.

[2] S. Ayrault, B. Jimenez, E. Garnier, M. Fedoroff, D. Jones, C. Loos-Neskovic, J Solid State Chem 1998, 141, 475-485.

[3] P. Nie, L. Shen, H. Luo, B. Ding, G. Xu, J. Wang, X. Zhang, J. Mat. Chem. A 2014, 2, 5852-5857.

[4] L. Wang, Y. Han, X. Feng, J. Zhou, P. Qi, B. Wang, Coord. Chem. Rev. 2016, 307, 361-381.

[5] Z.-D. Gao, Y. Qu, T. Li, N. K. Shrestha, Y.-Y. Song, Sci. Rep. 2014, 4, 6891.

[6] B. Kong, C. Selomulya, G. Zheng, D. Zhao, Chem. Soc. Rev. 2015, 44, 7997-8018.

[7] S. Ohkoshi, H. Tokoro, Acc Chem Res 2012, 45, 1749-1758.

[8] L. Catala, T. Mallah, Coord. Chem. Rev. 2017, 346, 32-61.

[9] W.-J. Xu, Z.-Y. Du, W.-X. Zhang, X.-M. Chen, CrystEngComm 2016, 18, 7915-7928.

[10] W. Li, Z. Wang, F. Deschler, S. Gao, R. H. Friend, A. K. Cheetham, Nat. Rev. Mater. 2017, 2, natrevmats201699.

[11] G. Kieslich, A. L. Goodwin, Mater. Horiz. 2017, 4, 362-366.

[12] N. L. Evans, P. M. M. Thygesen, H. L. B. Boström, E. M. Reynolds, I. E. Collings, A. E. Phillips, A. L. Goodwin, J. Am. Chem. Soc. 2016, 138, 9393-9396.

[13] M. Guo, H.-L. Cai, R.-G. Xiong, Inorg. Chem. Commun. 2010, 13, 1590-1598.

[14] W.-J. Xu, P.-F. Li, Y.-Y. Tang, W.-X. Zhang, R.-G. Xiong, X.-M. Chen, J. Am. Chem. Soc. 2017, 139, 6369-6375.

[15] W. Zhang, Y. Cai, R. Xiong, H. Yoshikawa, K. Awaga, Angew. Chem. Int. Ed. 2010, 49, 6608-6610.

[16] X. Zhang, X.-D. Shao, S.-C. Li, Y. Cai, Y.-F. Yao, R.-G. Xiong, W. Zhang, Chem. Comm. 2015, 51, 4568-4571. 
[17] A. G. Christy, Acta Crystallogr. B 1995, 51, 753-757.

[18] A. L. Goodwin, M. Calleja, M. J. Conterio, M. T. Dove, J. S. O. Evans, D. A. Keen, L. Peters, M. G. Tucker, Science 2008, 319, 794-797.

[19] A. B. Cairns, A. L. Thompson, M. G. Tucker, J. Haines, A. L. Goodwin, J. Am. Chem. Soc. 2012, 134, 4454-4456.

[20] T. Matsuda, J. E. Kim, K. Ohoyama, Y. Moritomo, Phys. Rev. B 2009, 79, 172302.

[21] S. Adak, L. L. Daemen, M. Hartl, D. Williams, J. Summerhill, H. Nakotte, J. Solid State Chem. 2011, 184, 2854-2861.

[22] A. L. Goodwin, K. W. Chapman, C. J. Kepert, J. Am. Chem. Soc. 2005, 127, 17980-17981.

[23] A. E. Phillips, G. J. Halder, K. W. Chapman, A. L. Goodwin, C. J. Kepert, J. Am. Chem. Soc. 2010, 132, 10-11.

[24] I. E. Collings, J. A. Hill, A. B. Cairns, R. I. Cooper, A. L. Thompson, J. E. Parker, C. C. Tang, A. L. Goodwin, Dalton Trans. 2016, 45, 4169-4178.

[25] J.-H. Her, P. W. Stephens, C. M. Kareis, J. G. Moore, K. S. Min, J.-W. Park, G. Bali, B. S. Kennon, J. S. Miller, Inorg. Chem. 2010, 49, 1524-1534.

[26] A. Plutecka, U. Rychlewska, Acta Cryst. C 2009, 65, m75-m77.

[27] M. Mączka, B. Bondzior, P. Dereń, A. Sieradzki, J. Trzmiel, A. Pietraszko, J. Hanuza, Dalton Trans. 2015, 44, 6871-6879.

[28] I. N. Flerov, M. V. Gorev, K. S. Aleksandrov, A. Tressaud, J. Grannec, M. Couzi, Mat. Sci. Eng. R. 1998, 24, 81-151.

[29] C. J. Howard, B. J. Kennedy, P. M. Woodward, Acta Cryst. B 2003, 59, 463-471.

[30] S. G. Duyker, J. A. Hill, C. J. Howard, A. L. Goodwin, J. Am. Chem. Soc. 2016, 138, 1112111123.

[31] P. M. Woodward, Acta Cryst. B 1997, 53, 32-43.

[32] S. A. Larrégola, J. A. Alonso, D. Sheptyakov, M. Algueró, A. Muñoz, V. Pomjakushin, J. C. Pedregosa, J. Am. Chem. Soc. 2010, 132, 14470-14480.

[33] S. A. Larrégola, J. A. Alonso, D. Sheptyakov, M. Algueró, A. Muñoz, V. Pomjakushin, J. C. Pedregosa, Inorg. Chem. 2011, 50, 5545-5557.

[34] S. A. Larrégola, J. A. Alonso, V. A. de la Peña-O'Shea, D. Sheptyakov, V. Pomjakushin, M. T. Fernandez-Díaz, J. C. Pedregosa, Inorg. Chem. 2014, 53, 5609-5618. 
[35] M. Retuerto, S. Skiadopoulou, M.-R. Li, A. M. Abakumov, M. Croft, A. Ignatov, T. Sarkar, B. M. Abbett, J. Pokorný, M. Savinov, D. Nuzhnyy, J. Prokleška, M. Abeykoon, P. W. Stephens, J. P. Hodges, P. Vaněk, C. J. Fennie, K. M. Rabe, S. Kamba, M. Greenblatt, Inorg. Chem. 2016, 55, 4320-4329. 


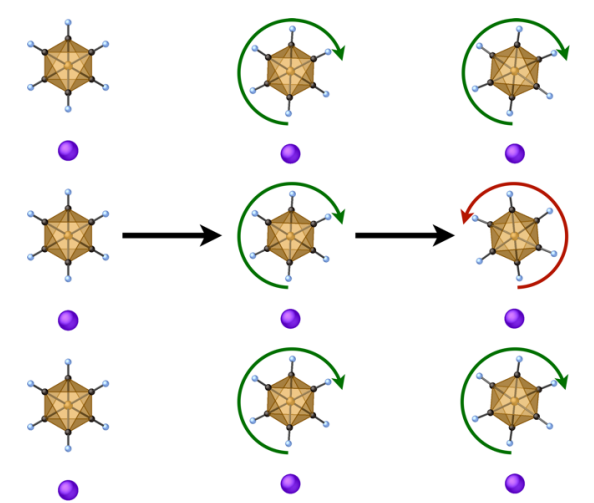

A different tilt: A reassessment of the symmetry of two cyanide-bridged perovskite analogues reveals that phase transitions in these materials arise from competition between two different tilt patterns. Tilt engineering of the hybrid materials provides a way to access combinations of distortions that are rare or impossible among the inorganic perovskites. 